\title{
Action Mechanism of Ginkgo biloba Leaf Extract Intervened by Exercise Therapy in Treatment of Benign Prostate Hyperplasia
}

\author{
Chiung-Chi Peng, ${ }^{1}$ Jia-Hong Liu, ${ }^{2,3}$ Chi-Huang Chang, ${ }^{2}$ Jin-Yuan Chung, ${ }^{1,4}$ Kuan-Chou \\ Chen, ${ }^{4}$ Kuang-Yu Chou, ${ }^{5}$ and Robert Y. Peng ${ }^{2}$ \\ ${ }^{1}$ Graduate Institute of Clinical Medicine, College of Medicine, Taipei Medical University, 250 Wu-Hsing Street, Taipei 11031, Taiwan \\ ${ }^{2}$ Research Institute of Biotechnology, Hungkuang University, 34 Chung-Chie Road, Shalu District, Taichung City 43302, Taiwan \\ ${ }^{3}$ Department of Urology, Shuang Ho Hospital, Taipei Medical University, 291 Zhongzheng Road, Zhonghe, Taipei 23561, Taiwan \\ ${ }^{4}$ Department of Urology, School of Medicine, College of Medicine, Taipei Medical University, 250 Wu-Hsing Street, Taipei 11031, Taiwan \\ ${ }^{5}$ Division of Urology, Department of Surgery, Shin Kong Wu Ho-Su Memorial Hospital, 95 Wen Chang Road, Taipei 111, Taiwan
}

Correspondence should be addressed to Kuan-Chou Chen; kc.chen416@msa.hinet.net; Kuang-Yu Chou; ky_chou@livemail.tw; and Robert Y. Peng; ypeng@seed.net.tw

Received 11 December 2012; Revised 26 January 2013; Accepted 26 January 2013

Academic Editor: Gerhard Litscher

Copyright (C) 2013 Chiung-Chi Peng et al. This is an open access article distributed under the Creative Commons Attribution License, which permits unrestricted use, distribution, and reproduction in any medium, provided the original work is properly cited.

Benign prostatic hyperplasia $(\mathrm{BPH})$, an imbalance between androgen/estrogen, overexpression of stromal, and epithelial growth factors associated with chronic inflammation, has become an atypical direct cause of mortality of aged male diseases. Ginkgo possesses anti-inflammatory, blood flow-enhancing, and free radical scavenging effects. Considering strenuous exercise can reduce $\mathrm{BPH}$ risks, we hypothesize Ginkgo + exercise (Ginkgo + Ex) could be beneficial to BPH. To verify this, rat BPH model was induced by s.c. $3.5 \mathrm{mg}$ testosterone (T) and $0.1 \mathrm{mg}$ estradiol (E2) per head per day successively for 8 weeks, using mineral oil as placebo. Cerenin ${ }^{\circledR} 8.33 \mu \mathrm{L} / 100 \mathrm{~g}$ was applied s.c. from the 10 th to the 13 th week, and simultaneously, Ex was applied ( $30 \mathrm{~m} / \mathrm{min}$, 3 times/week). In BPH, Ginkgo alone had no effect on T, $5 \alpha$-reductase, and dihydrotestosterone (DHT), but suppressed androgen receptor (AR), aromatase, E2 and estrogen receptor (ER), and the proliferating cell nuclear antigen (PCNA); Ex alone significantly reduced T, aromatase, E2, ER, AR, and PCNA, but highly raised DHT. While Ginkgo + Ex androgenically downregulated T, aromatase, E2, and ER, but upregulated DHT, AR, and PCNA, implying Ginkgo + Ex tended to worsen BPH. Conclusively, Ginkgo or Ex alone may be more beneficial than Ginkgo + Ex for treatment of BPH.

\section{Introduction}

Benign prostatic hyperplasia (BPH) and low urinary tract symptoms (LUTS) are quite common male diseases. The prevalence of $\mathrm{BPH}$ increases with aging [1] and has now become an atypical direct cause of mortality [2]. Biochemically, $\mathrm{BPH}$ is considered to be an imbalance between androgen/estrogen $[3,4]$, overexpression of stromal and epithelial growth factors, cytokines, and steroid hormones $[5,6]$. Pathologically, BPH is characterized by hyperplastic epithelial and stromal growth that emerge into numerous microscopic and macroscopic nodules in the prostate gland [7]. Tissue remodeling in the aging prostate $[8,9]$, stem cell defects [10], hypoxia [11], and chronic inflammation [12-16] or by many other factors is still obscure.

The clinical care for BPH usually involves $\alpha$-blockers, $5 \alpha$ reductase inhibitors (e.g., finasteride), and surgery therapy, or the combined treatment [17]. Currently, phytotherapeutic agents are emerging and frequently used as the complementary alternative treatment of BPH [18]. Other nonmedication agents include zinc, soy/tofu, selenium, vitamin E, and amino acids [19]. The main constituents of Ginkgo biloba leaf extract (for simplicity named "Ginkgo" herein) comprise Ginkgo flavone glycosides (biflavonols, quercetin; biflavones, sciadopitysin; and proanthocyanidins, procyanidin) and terpene lactone ginkgolides (ginkgolide A, B, C, 
and bilobalide) [20]. Ginkgo possesses antioxidant and antiinflammatory activity [21-24]. Ginkgo biloba leaf extract (Ginkgo) has been used for centuries in China for treating asthma and bronchitis. Currently, Ginkgo has been widely used to treat the cerebrovascular and the peripheral vascular insufficiency, neurosensory problems, and disturbances in vigilance, short-term memory, and other cognitive functions that are associated with dementias, ageing, and senility [25]. Many pharmacological and clinical studies have shown that the extract of Gingko causes two main actions: increase of blood flow in central and peripheral vessels and inhibition of platelet aggregation and free radical scavenging. Thus Gingko may be effective in cases of erectile dysfunction due to a decreased blood flow [26].

A review of Sea et al. (2009) highly supports a clinically significant, independent, and strong inverse relationship between exercise (Ex) and the development of BPH /LUTS [27]. Greater distances run per week may reduce BPH risk independent of BMI, $10 \mathrm{~km}$ performance, and diet [28].

The application of Ginkgo in treating BPH is still lacking. In view of the above mentioned beneficial biological activities of Gingko and Ex, we hypothesize that Ginkgo + Ex therapy as well could be beneficial to BPH. To verify this, we conducted this experiment and the relevant biochemical, immunological, and pathological parameters were examined and compared.

\section{Materials and Methods}

2.1. Chemicals. Testosterone, dihydrotestosterone (DHT), estradiol ELISA kits were provided by Cayman Chemical Co. (Michigan, USA). Free PSA and Total PSA assay kits were provided by Cusabio Biotech (Wuhan, China). Rat IL-1 ELISA development kit is product of PeproTech Co. (Rocky Hill, NJ, USA). Enhanced Chemiluminescence (ECL) system was provided by Merk Millipore Co. (Billerica, MA, USA). TEMED is a product of Bio-Rad Co. (Hercules, CA, USA). Protein Extraction Solution was provided by iNtRON Biotech. Co. (Kyungki-Do, Korea). The pharmaceutical preparation of androgen, Sustanon ${ }^{\circledR}$, is an inject testosterone medication provided by Schering-Plough Company (Kenilworth, NJ, USA), which in reality contains four testosterone esters. Each ampoule $(1 \mathrm{~mL})$ contains testosterone propionate $30 \mathrm{mg}$, testosterone phenylpropionate $60 \mathrm{mg}$, testosterone isocaproate $60 \mathrm{mg}$, and testosterone decanoate $100 \mathrm{mg}$. The overall androgenic potency in per $\mathrm{mL}$ of Sustanon ${ }^{\circledR}$ is equivalent to $176 \mathrm{mg}$ testosterone. The Ginkgo biloba leaf extract, Cerenin ${ }^{\circledR}$, was purchased from Dr. Willmar Schwabe Arzneimittel GmbH \& Co. (Karlsruhe, Germany). Ginkgo consisted of 24\% of Ginkgo flavone glycosides, mainly kaempferol and quercetin glucorhamnoside esters, and 6\% of the characteristic terpene lactones, the bilobalide, and ginkgolides, namely, A, B, C, and very small quantities of ginkgolide. The inject solution contained in per $\mathrm{mL} 3.5 \mathrm{mg}$ Ginkgo (240 mg/g flavonoids and $60 \mathrm{mg} / \mathrm{g}$ terpenoids), $30 \mathrm{mg}$ of $960 \mathrm{~mL} / \mathrm{L}$ ethanol, $40 \mathrm{mg}$ sorbitol, and $0.1 \mathrm{~mol} / \mathrm{L} \mathrm{NaOH}$. All other chemicals, not cited but used in this experiment, were of reagent grade provided by Wako Pure Chemical Co.
(Osaka, Japan). The sources of the antibodies used in this experiment were $5 \alpha$-reductase and androgen receptor (Santa Cruz Biotech Inc., Santa Cruz, CA, USA), estrogen receptor $\alpha$ $(\mathrm{ER} \alpha)($ Merk Millipore, Billerica, MA, USA), aromatase and proliferating cell nuclear antigen (PCNA) (Epitomics Inc., Burlingame, CA, USA), and $\beta$-actin from Novus Biologicals (Littleton, CO, USA).

2.2. Animal Grouping. This experiment was proved by the Institutional Animal Care and Ethic Committee of China Medical University (Taichung, Taiwan). Ninety-six SpragueDawley (SD) rats weighing approximately $280 \mathrm{~g}$ were purchased from BioLasco Animal Centre, Taiwan. The rats were housed in a controlled environment, 3 in each cage, with $12 \mathrm{~h} / 12 \mathrm{~h}$ light/dark cycle, at $28 \pm 1^{\circ} \mathrm{C}$ and under relative humidity $65-75 \%$. These rats were acclimated in such an environment for the first week. Then rats were divided into eight groups. The five controls involved Group 1, the normal control; Group 2, the BPH control; Group 3, the Ginkgo-only treated control; Group 5, the Ex-only control; and Group 7, the Ginkgo + Ex. The medication treated groups were Group 4, BPH treated with Ginkgo; Group 6, BPH treated with Ex; and Group 8, BPH treated with Ginkgo + Ex, each having 12 rats. The exercise groups were subjected to treadmill exercise protocol. The treatment started from week 10 to week 13 .

2.3. BPH Induction: The Hormone-Induced Rat BPH Model. The protocol to induce $\mathrm{BPH}$ was conducted according to Suzuki et al. (1994) with slight modification [29]. Briefly, in the beginning of week 2, all healthy controls (groups 1, 3, 5, and 7) were s.c. administered $20 \mu \mathrm{L}$ mineral oil/head/day as placebo. The BPH groups (groups 2, 4, 6, and 8) were s.c. administered a combined testosterone (Sustanon ${ }^{\circledR}$ ) $3.5 \mathrm{mg}$ with estradiol $0.1 \mathrm{mg}$ per head per day successively from week 2 to week 9 , that is, a total induction period of 8 weeks.

2.4. Ginkgo biloba Extract Administration Protocol. The dose of Cerenin ${ }^{\circledR}$ was calculated from the recommended dose for clinical human use, that is, $35 \mathrm{mg}$ ( $5 \mathrm{~mL}$ injection solution) i.v. for $60 \mathrm{~kg}$ adults. Thus in the beginning of week 10 immediately prior to the treatment experiment, a dose of Cerenin $^{\circledR} 8.33 \mu \mathrm{L} / 100 \mathrm{~g}$ was recommended daily for each Ginkgo medicated group. The whole treatment course sustained for 4 weeks (i.e., from week 10 to week 13).

2.5. Treadmill Exercise Training Protocol. For treadmill exercise training, the groups $5,6,7$, and 8 were first acclimated from week 8 to 9 , starting with $5 \mathrm{~min}, 10 \mathrm{~min}, 15 \mathrm{~min}$, and then 20 min per time per day. The formal exercise therapy was then started from week 10 until week 13, three times per week, $30 \mathrm{~min}$ per time at a speed of $30 \mathrm{~m} / \mathrm{min}$ on a motorized rodent treadmill (Fortelice International Co., Ltd., Taipei, Taiwan).

2.6. Blood and Tissue Collection. At the end of week 13, blood samples were withdrawn from the abdominal aorta under ether anesthesia. The blood was collected and centrifuged at $3000 \times \mathrm{g}$ for 15 minutes to separate the serum. The prostates 
were excised, immediately frozen with liquid nitrogen and stored in $-80^{\circ} \mathrm{C}$ or fixed by immersion with $10 \%$ formalin in PBS (pH 7.4).

2.7. Hematoxylin-Eosin (HE) and Sirius Red Staining. The prostates were fixed by immersion in $10 \%$ formalin-PBS $(\mathrm{pH}$ 7.4) at $4^{\circ} \mathrm{C}$ for $24 \mathrm{~h}$ and processed for paraffin embedding. Paraffin sections were dewaxed in xylene and rehydrated in a series of ethanol washes. The nuclei of these specimens were subjected to hematoxylin-eosin stain. Otherwise, the collagen content was stained with Sirius Red (Sigma-Aldrich Co., MO, USA) [30].

2.8. Enzyme-Linked Immunosorbent Assay (ELISA). Serum levels of testosterone, estradiol, dihydrotestosterone (DHT), prostate-specific antigen (PSA), and IL-1 were measured by the ELISA kits. All protocols were performed by following the manufacturer's instruction. The EZ Read 400 Microplate Reader used was a product of Biochrom Co. (Cambridge, UK) [30].

2.9. Immunohistochemical (IHC) Staining. The protein expression of androgen receptor and proliferating cell nuclear antigen were analyzed by IHC according to previous protocol cited [30]. Quantitative analysis was performed using an Image-Pro Plus (Meyer Instruments, USA) analysis system. The integrated optical density (IOD) was measured. The sum of the IOD was obtained with the mean value calculated.

2.10. Western Blotting. Levels of $5 \alpha$-reductase, estrogen receptor $\alpha$, and aromatase were analyzed. Briefly, frozen prostate tissue samples (approximately $100 \mathrm{mg}$ ) were homogenized with the homogenizer (T10 basic, The IKA Company, Germany) in $1 \mathrm{~mL}$ of Pro-PREP lysis buffer $(\mathrm{pH}$ 7.2). The homogenate was centrifuged at $12000 \times \mathrm{g}$ for $20 \mathrm{~min}$ at $4^{\circ} \mathrm{C}$, and the supernatant was collected as tissue sample lysate. The sample lysates were heated at $100^{\circ} \mathrm{C}$ for $10 \mathrm{~min}$ before loading and separated on precasted $7.5 \%$ SDS-PAGE. The proteins were electrotransferred onto the PVDF membrane in transfer buffer for $1 \mathrm{~h}$.

The nonspecific binding to the membrane was blocked for $1 \mathrm{~h}$ at room temperature with $5 \%$ nonfat milk in TBS buffer. The membranes were then incubated for $16 \mathrm{~h}$ at $4^{\circ} \mathrm{C}$ with various primary antibodies. After extensive washing in TBS buffer, the membranes were then incubated with secondary antibody in blocking buffer containing 5\% nonfat milk for $1 \mathrm{~h}$ at room temperature. Membranes were then washed with TBS buffer and the signals were quantified using the Luminescent Image Analyzer LAS-4000 (Fujifilm, Tokyo, Japan). $\beta$-actin was used as the reference protein [30].

2.11. Statistical Analysis. Data obtained in the same group were treated with ANOVA and Duncan's multiple range tests with computer statistical software SAS 9.0 (SAS Institute Inc., Cary, NC, USA). Different letters indicate significant differences at $P<0.05$.

\section{Results}

3.1. BPH Reduced the Body Weight but Raised the Ratio of Prostate Weight to Body Weight. Starting from the original body weight (275 to $300 \mathrm{~g}$ ), the body weight of all groups was seen increasing steadily from week 0 to week 2 (week 1 for acclimation) (Figure 1(a)). When induced with $\mathrm{BPH}$ at week 2 and with induction maintained daily until week 9 , all control groups showed substantive increase of body weight to range within $455.5 \pm 36.2 \sim 524.4 \pm 35.2 \mathrm{~g}$ at week 13 . As contrast, in all BPH groups no apparent body weight increase was found; all remained in range $331.0 \pm 44.2 \sim 356.9 \pm 34.2 \mathrm{~g} / \mathrm{rat}$ until week 13 (Figure 1(a)). The ratio prostate to body weight remained within $0.004 \sim 0.005$ in all normal groups but were raised to $0.008 \sim 0.009$ in all BPH rats (Figure $1(\mathrm{~b})$ ).

3.2. Microscopic Pathological Examination. Microscopically, in the normal prostates the acini were lined by columnar epithelial cells and the lumens were filled with eosinophilic secretion. Regular acini and alignment were apparently perceivable (Figure 2(a), upper panel). In the BPH prostates, mild epithelial hyperplasia and irregular acinar shape with villous projections were clearly perceived. The epithelial hyperplasia budding out with intraepithelial vacuoles was very apparent, and in this region some epithelial cells indicated loss of polarity (Figure 2(a) lower panel). Statistically, the pathological incidence rate (rat number per group) of prostate hyperplasia was $0.00,0.73,0.00$, and 0.18 for the four groups without exercise, that is, the normal control, $\mathrm{BPH}$ control, Ginkgo control, and BPH + Ginkgo, respectively. When treated with exercise, the incidence rates were apparently shifted to $0.00,0.00,0.00$, and 0.18 , respectively, for Ex control, BPH + Ex, Ginkgo + Ex, and BPH + Ginkgo + Ex. Usually $\mathrm{BPH}$ is accompanied with inflammation. The incidence rate of inflammation was $0.00,0.64,0.08$, and 0.18 , for the normal control, BPH control, Ginkgo control, and BPH + Ginkgo, respectively. After exercise training, the incidence rate of inflammation was improved to give $0.00,0.46,0.25$, and 0.55 , respectively, for Ex control, $\mathrm{BPH}+\mathrm{Ex}$, Ginkgo + Ex, and $\mathrm{BPH}$ + Ginkgo + Ex (Drs. Chen T.-Y. and Lee K.-H., Diagnostic Laboratory of Laboratory Rodents, National Laboratory Animal Center (NLAC), Taipei, Taiwan), implying the fact that Ginkgo was beneficial to curing $\mathrm{BPH}$, and also effective to suppress the inflammation status. The Sirius Red stain revealed huge amount of collagen deposition occurring in BPH group (Figures 2(b) and 2(c)), apparently localized in the interstitial tissues and intracellular cytoplasm. Ginkgo alone seemed to have alleviated the majority of these pathologically adverse effects. Exercise alone improved it to some extent only comparable to the effect with Ginkgo + Ex (Figures 2(b) and 2(c)). Amazingly, the normal Ex groups also revealed some collagen deposition (Figures 2(b) and 2(c)).

3.3. Serum Total Prostate-Specific Antigen ( $t$-PSA) in BPH Was Upregulated by Ginkgo, but Suppressed by Exercise Training. The normal total PSA level of the control and the BPH groups were ranging within $44 \pm 8 \mathrm{ng} / \mathrm{mL}$ and $55 \pm 5 \mathrm{pg} / \mathrm{mL}$ (Figure 3(a)). Ginkgo elevated the levels in both the Ginkgo 


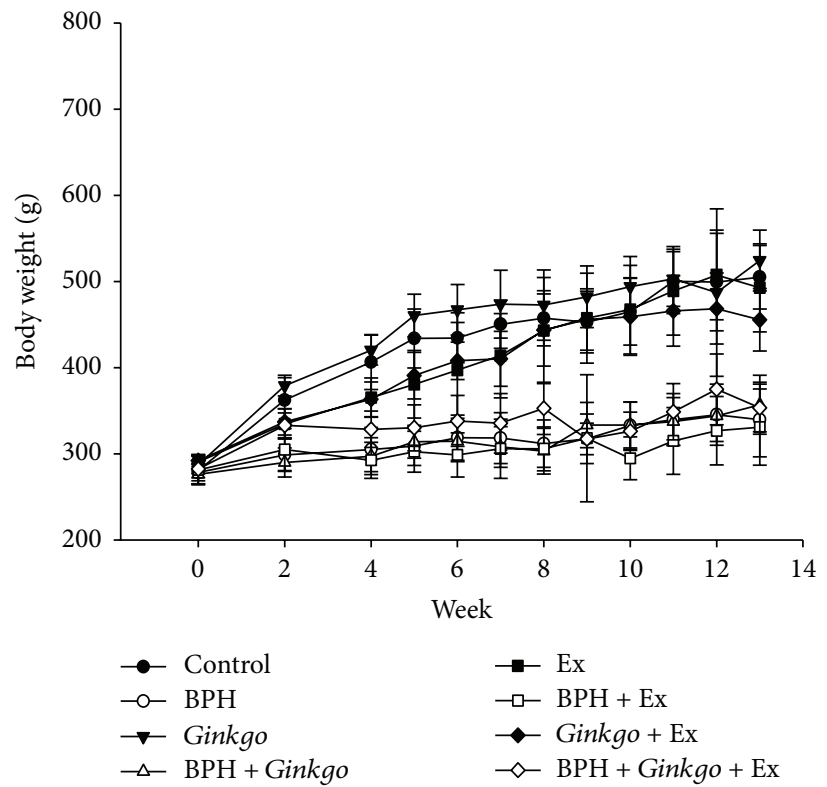

(a)

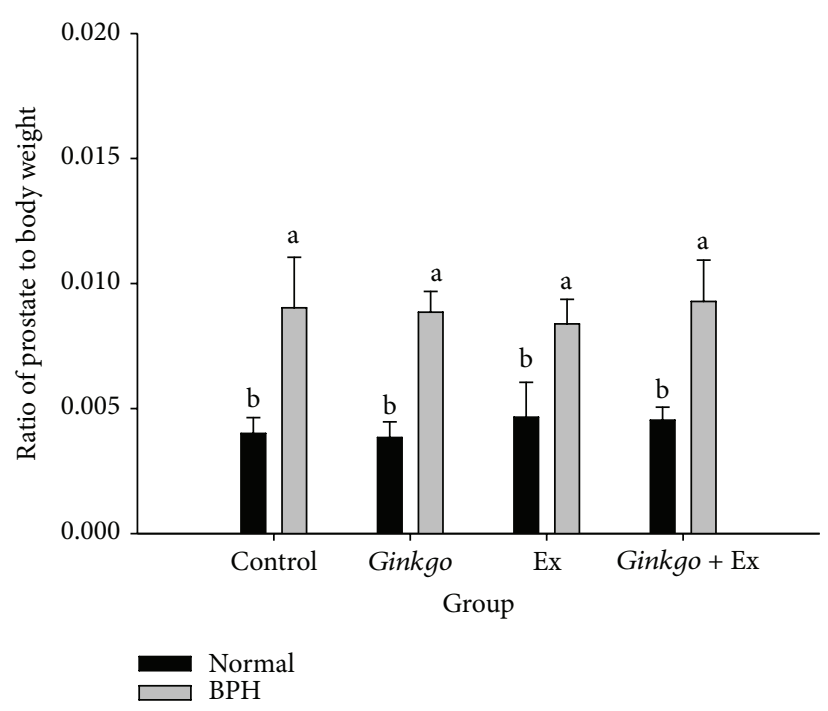

(b)

FIGURE 1: Duration-dependent variation of body weight (a) and the ratio prostate to body weight among all groups (b). Week 0-week 1: acclimation. Week 2-week 8: BPH induction period. Week 9: exercise pretraining. Week 10-week 13: receiving treatment. The grouping of rats was described in the text. (12 rats in each group, $P<0.05) \mathrm{BPH}$ : benign prostate hyperplasia. Ex: exercise.

and the $\mathrm{BPH}+$ Ginkgo groups significantly to $57 \pm 6 \mathrm{pg} / \mathrm{mL}$ and $68 \pm 9 \mathrm{pg} / \mathrm{mL}$. As contrast, exercise significantly reduced the total PSA levels in both the Ex and BPH + Ex groups. Conversely, in the Ginkgo + Ex group, both levels remained unchanged when compared with the normal and $\mathrm{BPH}$ control groups (Figure 3(a)).

3.4. Free PSA/Total PSA Ratio Was Suppressed by Ginkgo, Exercise, and the Combined Therapy. The free PSA to total PSA ratio ( $f$-PSA/t $t$-PSA) in normal controls ranged within $1.03 \pm 0.12 \%$, and that of BPH within $0.86 \pm 0.10 \%$. Ginkgo, exercise, and Ginkgo + Ex all significantly reduced the levels to ranges within $0.48 \sim 0.68 \mathrm{pg} / \mathrm{mL}$ (Figure 3(b)). No apparent difference was found for any treated group, indicating the effect of Ginkgo to be comparable to exercise alone. However in the combined therapy of Ginkgo + Ex, no better effect was further found (Figure 3(b)); suggestively, the action mechanism of Ginkgo and exercise probably via the same pathway.

3.5. The Serum Level of Testosterone Was Not Affected by Ginkgo Alone, but Improved Slightly by Exercise and the Combined Therapy. The serum level of testosterone in the normal control maintained at a level of $806.8 \pm 175.6 \mathrm{pg} / \mathrm{mL}$. While in the BPH control, it was apparently raised to $1494.1 \pm$ $202.1 \mathrm{pg} / \mathrm{mL}$. Exercise alone and Ginkgo + Ex seemed to have only slightly yet significantly suppressed these testosterone levels (Figure 4(a)).

3.6. Ginkgo Alone Did Not Affected DHT, However Exercise and the Combined Therapy Significantly Raised DHT Level in BPH Groups. Ginkgo alone did not affect the prostatic DHT levels in both the normal and BPH groups. Alternatively, exercise and the combined therapy significantly elevated the prostatic DHT levels to reach $1420.4 \pm 58.8$ and $1338.7 \pm$ $49.8 \mathrm{pg} / \mathrm{mL}$ in $\mathrm{BPH}$ groups, respectively (Figure $4(\mathrm{~b})$ ). The DHT levels in the normal Ginkgo + Ex group also increased $(844.64 \pm 39.1 \mathrm{pg} / \mathrm{mL})$ compared with normal control $(530.8 \pm 68.3 \mathrm{pg} / \mathrm{mL}$ ) (Figure 4(b)).

As contrast, Western blotting revealed $5 \alpha$-reductase to be totally unaffected by any of these specific therapies (Figure $4(\mathrm{c})$ ).

3.7. AR Highly Upregulated by BPH Was Separately Downregulated by Ginkgo and Ex Alone, yet Further Upregulated by Ginkgo + Ex Therapy. Immunohistochemical stain showed prostatic androgen receptors were highly upregulated in $\mathrm{BPH}$ group (IOD $3.15 \pm 0.32 \%$ ) (Figures 5(a) and 5(b)). Ginkgo or exercise was shown able to suppress most parts of the upregulated AR (IOD $2.52 \pm 0.04 \%, 1.74 \pm 0.23 \%$ ). However strangely, Ginkgo + Ex further upregulated the level of AR in BPH group (IOD $3.97 \pm 0.48 \%$ ) (Figures 5(a) and 5(b)).

3.8. BPH Highly Upregulated Serum Estradiol Which Was Suppressed by Ginkgo, Ex, and Ginkgo + Ex. Alternatively, the estradiol level of the normal control ranged within $37.5 \pm$ $5.6 \mathrm{pg} / \mathrm{mL}$, and that of BPH control within $60.2 \pm 4.0 \mathrm{pg} / \mathrm{mL}$. All three treatments were shown to improve to $23.1 \pm 3.1$, $31.6 \pm 2.0$, and $43.4 \pm 8.6 \mathrm{pg} / \mathrm{mL}$, respectively, by Ginkgo, Ex, and Ginkgo + Ex (Figure 6(a)). BPH Control, Completely Ameliorated by Ginkgo, and Partially 


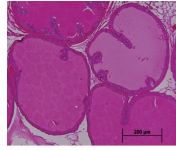

Normal

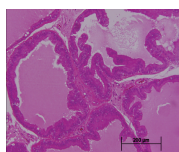

BPH

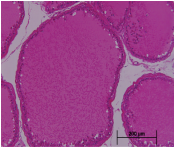

Ginkgo

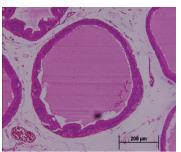

$\mathrm{BPH}+$ Ginkgo

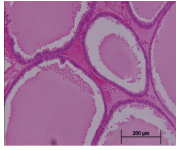

Ex

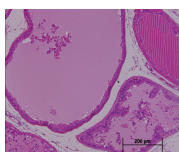

$\mathrm{BPH}+\mathrm{Ex}$

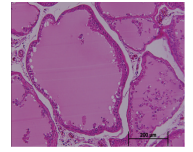

Ginkgo + Ex

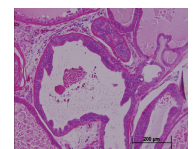

$\mathrm{BPH}+$ Ginkgo +

Ex

(a)

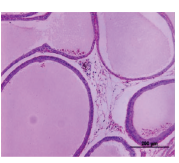

Normal

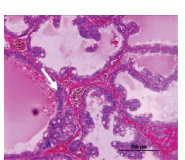

$\mathrm{BPH}$

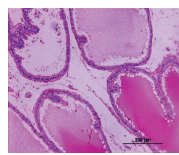

Ginkgo

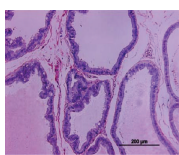

$\mathrm{BPH}+$ Ginkgo

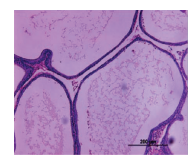

Ex

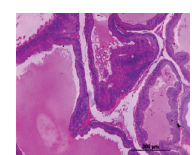

$\mathrm{BPH}+\mathrm{Ex}$

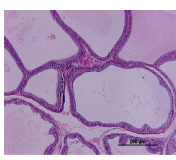

Ginkgo + Ex

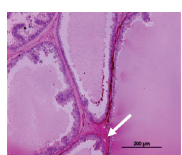

$\mathrm{BPH}+$ Ginkgo +

Ex

(b)

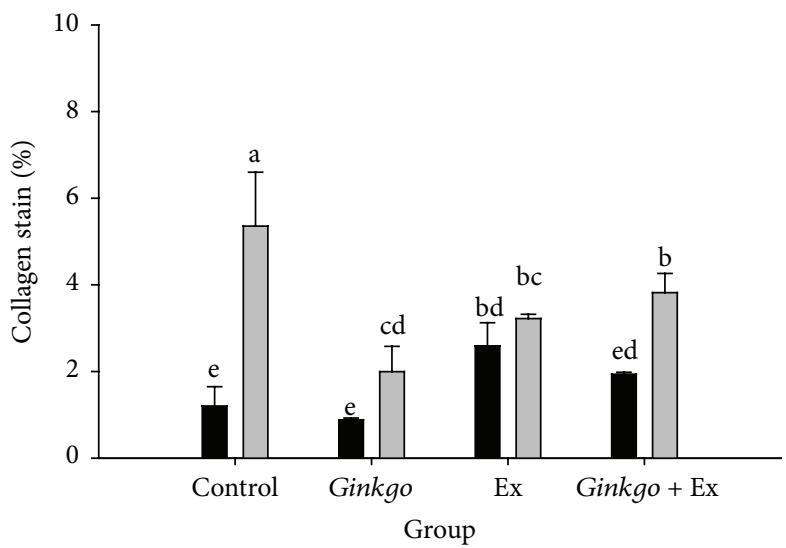

Norma

$\mathrm{BPH}$

(c)

FiguRE 2: Hematoxylin-eosin stain (a) and Sirius Red stain of collagen deposition (b) of prostatic tissues of the normal controls (upper panel), and the BPH groups (lower panel) $(\times 200)$; and the quantification of collagen deposition (c). (Upper panels: normal, Gingko control, Ex control, and Gingko + Ex control; lower panels: BPH control, BPH + Gingko, BPH + Ex, and BPH + Gingko + Ex.) Data were collected and statistically treated with ANOVA and Duncan's multiple range tests $(n=3)$. Different letters indicate significant difference between groups $(P<0.05)$. BPH: benign prostate hyperplasia. Gingko: the Gingko biloba leaf extract. Ex: exercise.

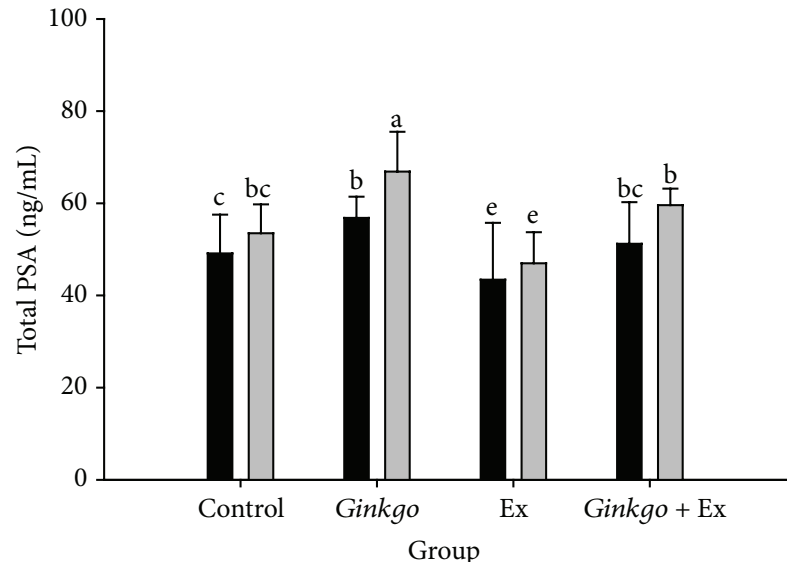

Normal

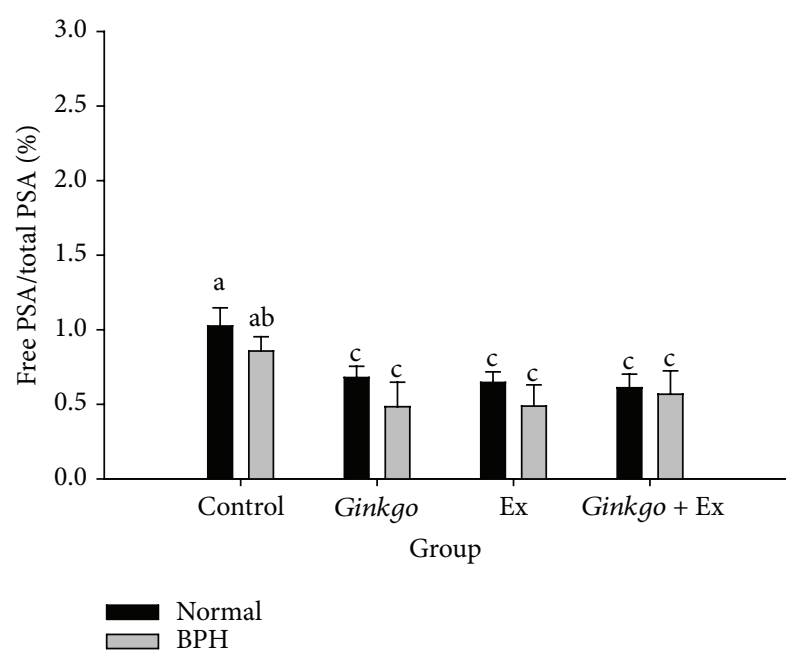

(b)

FIgURE 3: Serum level of total-PSA (a) and the percent ratio free-to-total PSA (b), of the normal controls and the BPH groups when treated with Gingko, Ex, and Ginkgo + Ex. Data were collected and statistically treated with ANOVA and Duncan's multiple range tests $(n=3)$. Different letters indicate significant difference between groups $(P<0.05)$. PSA: prostate-specific antigen. BPH: benign prostate hyperplasia. Ex: exercise. 


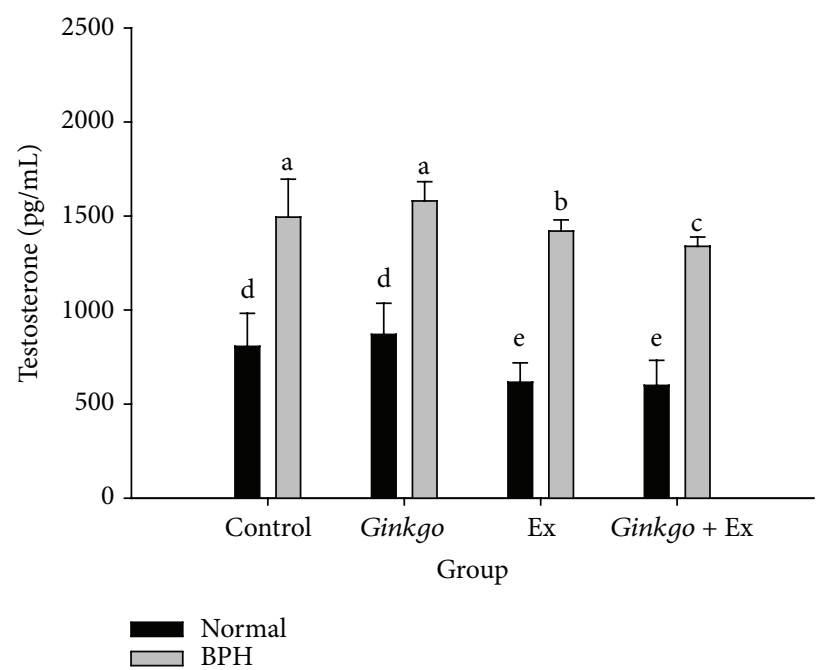

(a)

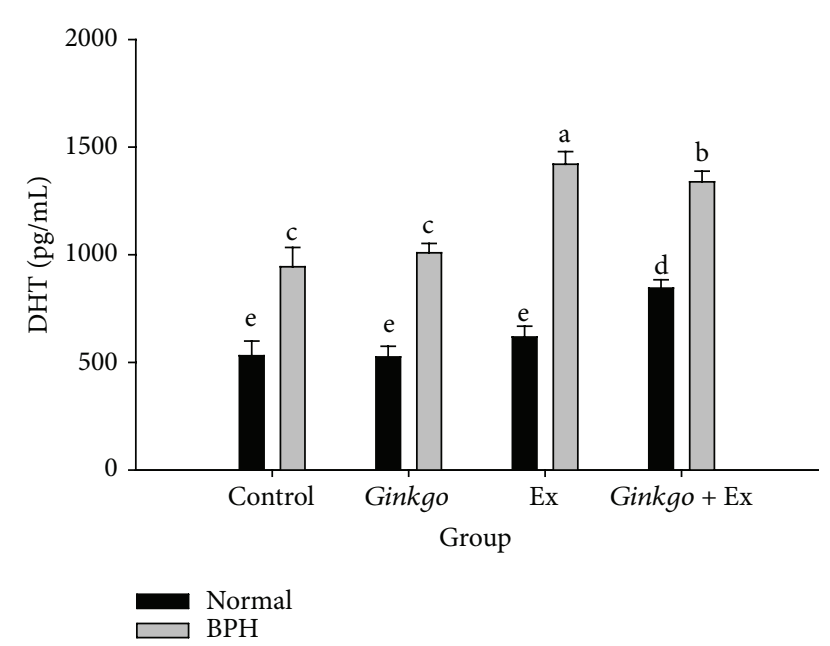

(b)
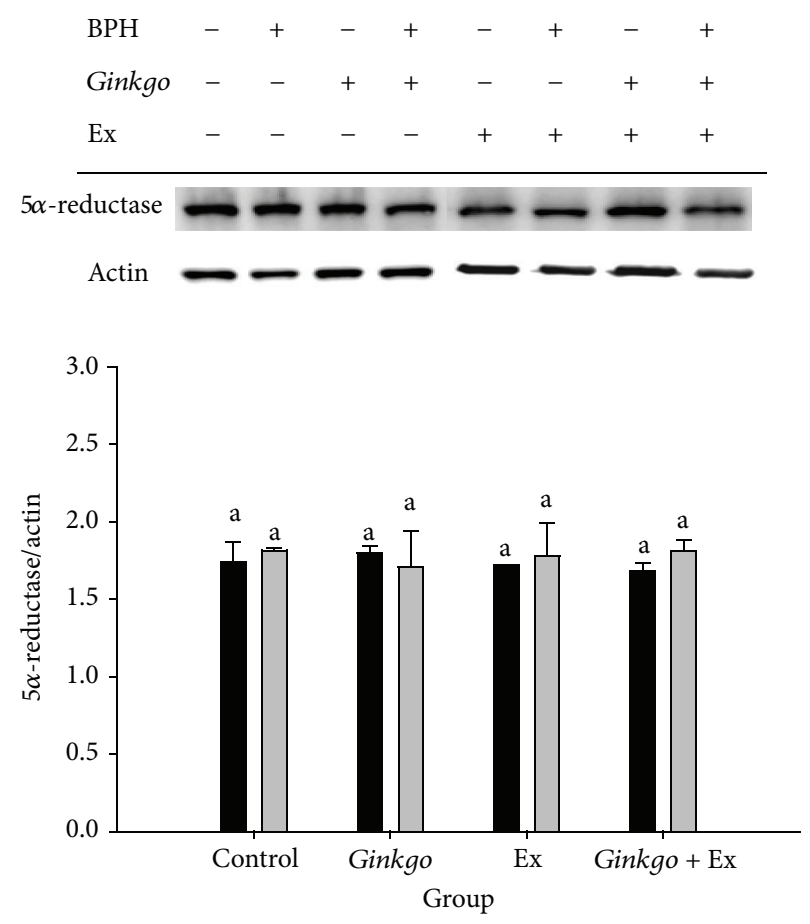

Normal

$\mathrm{BPH}$

(c)

Figure 4: Serum levels of Testosterone (a), DHT (b), and the Western blotting of $5 \alpha$-reductase (c) in prostates of the normal controls and the $\mathrm{BPH}$ groups. $\beta$-actin was used as the reference constitutive protein. Data were collected and statistically treated with ANOVA and Duncan's multiple range tests $(n=3)$. Different letters indicate significant difference between groups $(P<0.05)$. DHT: dihydrotestosterone. BPH: benign prostate hyperplasia. Ex: exercise. 


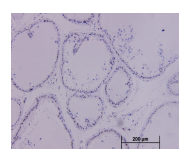

Normal

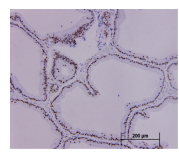

$\mathrm{BPH}$

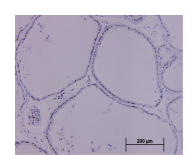

Ginkgo

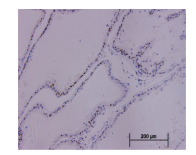

$\mathrm{BPH}+$ Ginkgo

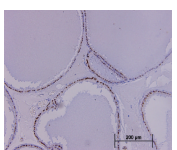

Ex

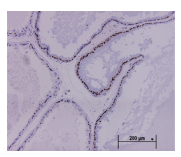

$\mathrm{BPH}+\mathrm{Ex}$

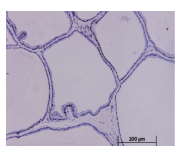

Ginkgo + Ex

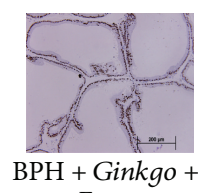

Ex
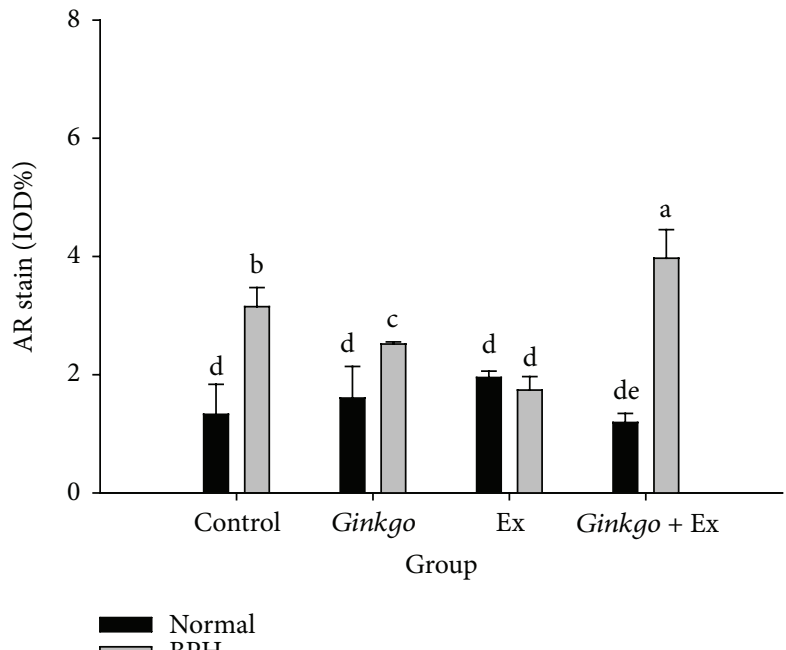

$\mathrm{BPH}$

(a)

(b)

FIGURE 5: Immunohistochemical stain of AR (a), and its quantification (b) in the prostate tissues of the normal controls and the BPH groups. (Upper panels: normal, Gingko control, Ex control, and Gingko + Ex control; lower panels: BPH control, BPH + Gingko, BPH + Ex, and BPH + Gingko + Ex.) Data were collected and statistically treated with ANOVA and Duncan's multiple range tests $(n=3)$. Different letters indicate significant difference between groups $(P<0.05)$. AR: androgen receptor. BPH: benign prostate hyperplasia. Ex: exercise.

Alleviated by Ex Alone and Ginkgo + Ex. The levels of estrogen receptor in all normal groups were not affected by any of the three treatments. Conversely, the ER highly upregulated in the BPH group was significantly downregulated by all the three therapies. Ginkgo in this case completely alleviated the upregulated level of ER (Figure 6(b)). Alternatively, exercise and the combined therapy seemed to be less effective than Ginkgo alone (Figure 6(b)).

3.10. Ginkgo Suppressed the Aromatase Level of BPH to Better Than Either Ex or the Combined Therapy. Similarly, BPH upregulated prostatic aromatase, Ginkgo completely alleviated, and exercise alone and Ginkgo + Ex were shown able to partially ameliorate the upregulation of prostatic aromatase. Interestingly, the levels of aromatase in all normal groups were unchanged (Figure 6(c)).

3.11. Prostate IL-1, Highly Upregulated in BPH, Was Ameliorated by Any of the Therapies. In prostate of $\mathrm{BPH}$, the level of IL-1 was significantly raised to $5.3 \pm 1.2 \mathrm{ng} / \mathrm{mL}$, which was completely ameliorated by all therapies (Figure 7), implying Ginkgo, exercise, and the combined therapy to act as effective anti-inflammatory agents.

3.12. PCNA Raised in BPH Was Ameliorated by Ginkgo, Exercise, and the Combined Treatments. Either Ginkgo or exercise was found to have effectively suppressed the upregulated PCNA in BPH group (Figure 8), apparently showing their promising antiproliferative effect. Astonishingly, the PCNA was insuppressible by the combined therapy (Figure 8). Data were slightly inconsistent with the pathological findings (NLAC).

\section{Discussions}

The normal and all controls showed normal growth increasing rate, conversely the growth rates of all $\mathrm{BPH}$ victims were severely retarded (Figure 1(a)). In reality, the body weight and prostate weight can be greatly affected by hormonal status [29]. The castrated male Wistar rats, the castrated + T, and the castrated $+\mathrm{T}+\mathrm{E} 2$ showed body weight at age 11 weeks $419.6 \pm$ $26.2,438.0 \pm 27.3$, and $379.6 \pm 26.4 \mathrm{~g}$, respectively, comparing to the normal control body weight $478.6 \pm 59.9 \mathrm{~g}$ [29]. The body weight retardation in the $\mathrm{BPH}$ groups was apparently perceivable. All experimental $\mathrm{BPH}$ SD rats retained their body weight within $331.0 \pm 44.2 \sim 356.9 \pm 34.2 \mathrm{~g}$ at week 13 (Figure 1(b)), evidencing the main antigrowth effect exerted by the combined treatment of $\mathrm{T}+\mathrm{E} 2$.

The effect of Ginkgo alone or any of the combined therapy was more or less effective, but limited to some aspects regarding the amelioration of pathological damages in prostates, like the lining up acini by columnar epithelial cells, eosinophilic secretion, epithelial hyperplasia, and deformed acinar shape (Figure 2(a), lower panel). Much of the literatures have indicated many benefits of Ginkgo; however most of which were limited only to its antioxidant and anti-inflammatory effects [21-24, 31]. To our belief, we are the first who report Ginkgo to be beneficial to BPH. Ginkgo was shown to be the most effective with respect to suppressing the intracellular cytoplasmic collagen deposition in the interstitial tissues (Figures 2(b) and 2(c)). Tissue from men with lower urinary tract symptoms was significantly stiffer $(P=0.00160)$ with significantly higher collagen content $(P=0.0038)$ and lower granularity than that from men without lower urinary tract symptoms (American Urological Association symptom index 8 or greater versus 7 or less) [32]. Thus, fibrosis can be a factor contributing to lower urinary tract symptom etiology. 

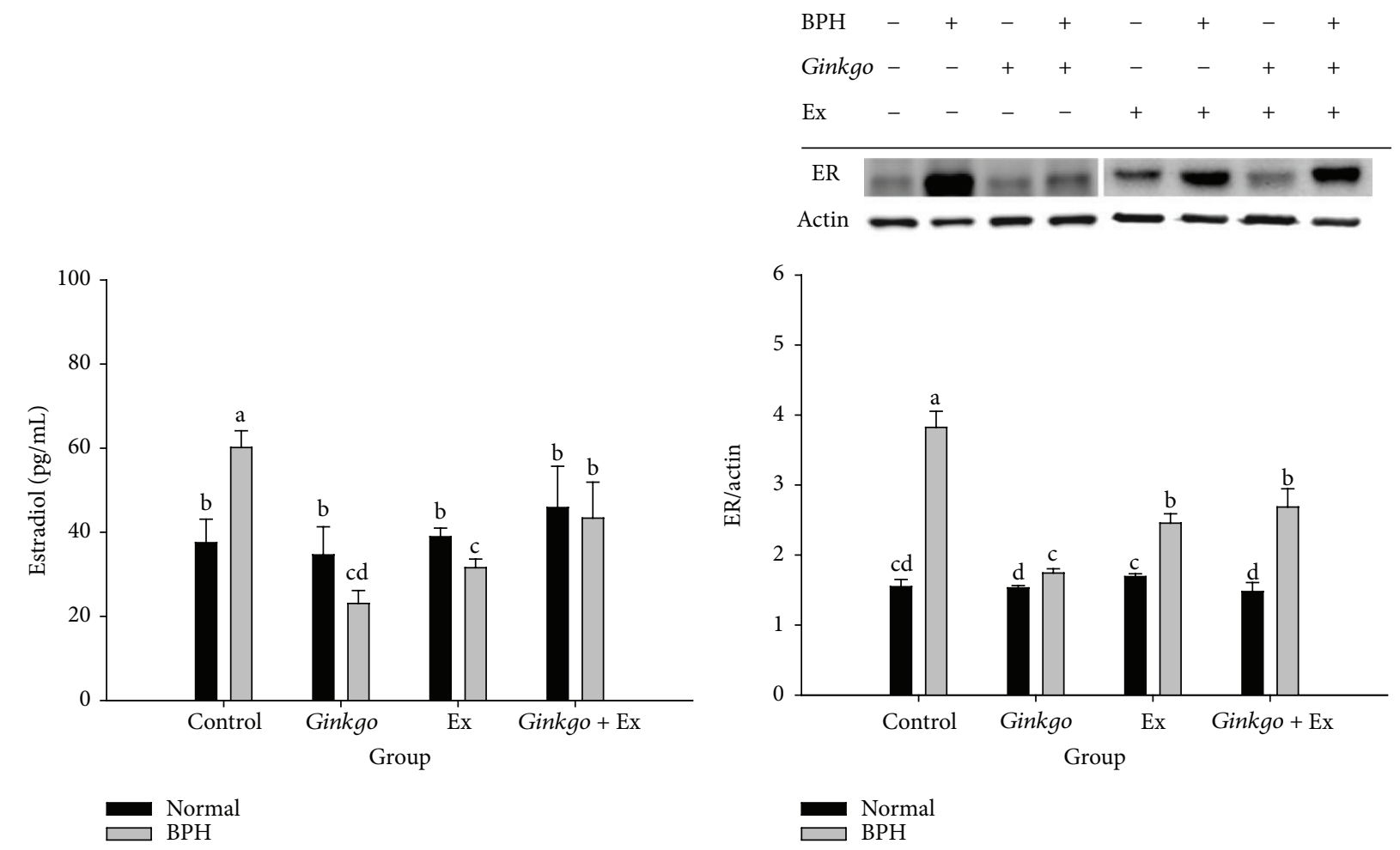

(a)

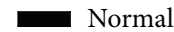

$\square \mathrm{BPH}$

(b)
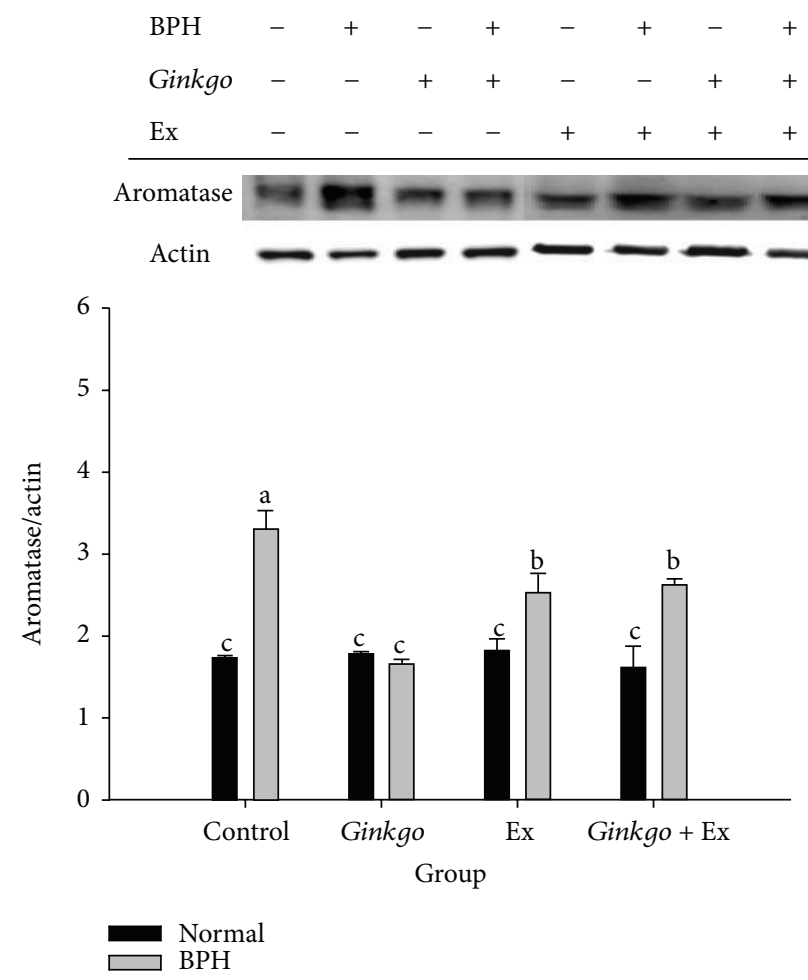

(c)

Figure 6: Serum levels of Estradiol (a), the Western blotting of ER ((b) upper) and its quantification ((b) lower), and the Western blotting of aromatase ((c) upper) and its quantification ((c) lower) in the prostates of different groups. $\beta$-actin was used as the reference constitutive protein. Data were collected and statistically treated with ANOVA and Duncan's multiple range tests $(n=3)$. Different letters indicate significant difference between groups $(P<0.05)(n=3)$. ER: estrogen receptor. Ex: exercise. 


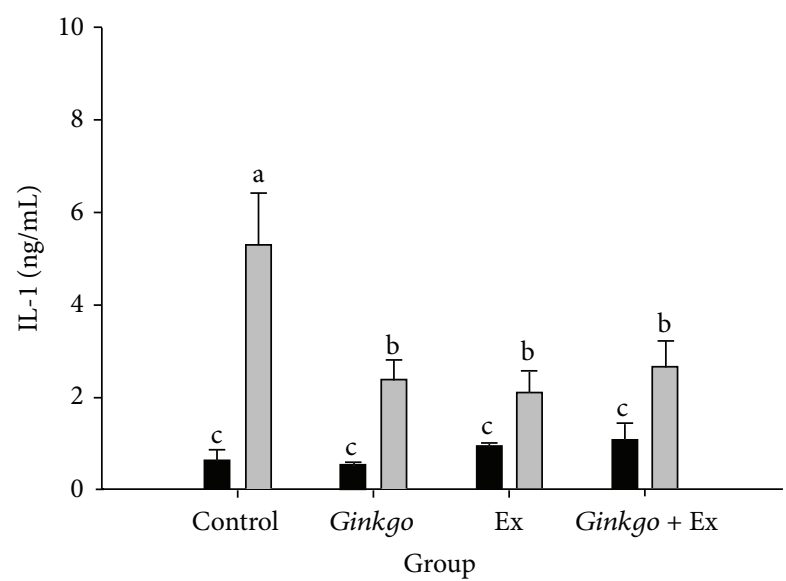

Normal
$\mathrm{BPH}$

FIGURE 7: Levels of prostatic tissue interleukin-1 (IL-1) affected by different therapies. Data were collected and statistically treated with ANOVA and Duncan's multiple range tests $(n=3)$. Different letters indicate significant difference between groups $(P<0.05)$.

Diagnostically, prostate-specific antigen (PSA) and the ratio of free-to-total PSA are widely used as the tumor markers, but the effect of exercise on these parameters is unclear. Ratio of free-to-total PSA was equally improved by Ginkgo, exercise, and Ginkgo + Ex (Figure 3(b)). Literature indicated that the free-to-total PSA ratio was significantly lower statistically in master athletes compared with recreational athletes, but is not clinically significant [33]. Nonetheless, the free-tototal PSA ratio can be affected by long-term athletic training, which could be rather important when evaluating athletes with prostate-related disorders [33].

The testosterone levels in groups $\mathrm{BPH}+\mathrm{Ex}$ and $\mathrm{BPH}+$ Ginkgo + Ex were seen significantly lower than those in the $\mathrm{BPH}$ control. Speculatively, the highly upregulated DHT in these two groups implied BPH possibly incurable by these treatments. AR was seen more highly expressed in $\mathrm{BPH}+$ Ginkgo + Ex group (Figure 5), while the $5 \alpha$-reductase in all groups remaining at comparable levels, a fact evidently suggesting that exercise and Ginkgo + Ex probably were only capable of enhancing the activity but incapable of affecting the quantity of $5 \alpha$-reductase (Figure $4(\mathrm{c})$ ). Suzuki et al. demonstrated that the values of both the $V_{\max }$ and the $K_{m}$ for nuclear $5 \alpha$-reductase in the rat dorsal lateral prostate were enhanced by treatment with T + E2 [29]. Thus our findings were rather consistent with Suzuki et al. (1994) [29].

Testosterone is biotransformed into estradiol-17 $\beta$ by action of aromatase. The prostate is an estrogen target tissue and estrogens directly and indirectly affect growth and differentiation of prostate [2]. BPH highly elevated the levels of aromatase, estradiol, and ER (Figure 6). Estrogens and selective estrogen receptor modulators have been shown to promote or inhibit prostate proliferation, signifying potential role of BPH and LUTS [2]. Aromatase, estradiol, and ER upregulated in $\mathrm{BPH}$ were suppressed by all the three treatments, while Ginkgo showed the most promising effect (Figure 6).

Ginkgo has been shown to exhibit estrogenic and antiestrogenic activities depending on the E2 and Ginkgo concentration, via ER-dependent and ER-independent pathways [34]. Ginkgo reduced the E2 levels by stimulating the E2 metabolism and inhibiting E2 synthesis [34]. As BPH usually is accompanied with inflammation $[12,14]$, results apparently pointed to the rescuing effect of these three therapies.

Clinical studies have revealed a close relationship between inflammation and prostate disease $[12,14]$. Much of literatures showed more than $92 \%$ incidences of inflammatory lesions in prostate tissue in $\mathrm{BPH}[15,16]$. IL- 1 is a paracrine inducer of FGF7, a key epithelial growth factor in $\mathrm{BPH}$ [35]. In BPH, IL-1 can be upregulated to induce FGF7, which in turn leads to further epithelial growth and increases IL-1 secretion, establishing the so-called "Double Paracrine Loop" [35]. Ginkgo, Ex, and Ginkgo + Ex all effectively alleviated the elevation of IL-1 (Figure 7). As well known, such anti-inflammatory effect has been well cited [21-24, 31].

Level of PCNA was lowered in Ginkgo and Ex groups comparing to the BPH control. Conversely, the PCNA level in Ginkgo + Ex remained unaffected at a level as high as the BPH control (Figure 8(b)).

Literature indicated that the pathogenesis of $\mathrm{BPH}$ could be caused by high proliferating rate and low apoptosis rate of hyperplasia epithelium [36]. Recent studies by AlonsoMagdalena et al. demonstrated $\mathrm{BPH}$ is not a disease of prostatic stroma proliferation but rather of accumulation of mesenchymal-like cells derived from the prostatic epithelium and the endothelium [9]. No evidence of proliferation was found in the stroma but in the epithelium of some ducts; $0.7 \%$ of the basal and $0.4 \%$ of the luminal cells were positive for the nuclear antigen Ki67 and the PCNA [9].

In summary, BPH tended to retard growth and increase organ weights of prostate. Although Ginkgo, exercise, and Ginkgo + Ex were all effective in alleviating collagen deposition, the combined Ginkgo + Ex was the most ineffective. Testosterone was unaffected by Ginkgo but significantly suppressed by Ex and Ginkgo + Ex. The levels of $5 \alpha$-reductase were totally unaffected by all three treatments. Ginkgo alone did not affect DHT level, but Ex and Ginkgo + Ex highly stimulated DHT. Ginkgo and Ex downregulated AR, but Ginkgo + Ex highly upregulated the level to higher than the BPH control. The upregulated E2 was suppressed by all three therapies. The highly upregulated PCNA in BPH was significantly and separately suppressed by Ginkgo and Ex, but unalleviated by Ginkgo + Ex. Results that underlie both Ginkgo and exercise were effective, but the combined therapy was ineffective for $\mathrm{BPH}$ treatment. The action mechanisms of Ginkgo and exercise in treating $\mathrm{BPH}$ are summarized in Figure 9.

\section{Conclusion}

In BPH, Ginkgo acts as an antiandrogenic (regarding the DHT level), an anti-AR, an aromatase inhibitor, and a potent anti-ER and a strong antiestrogenic (regarding the estradiol 


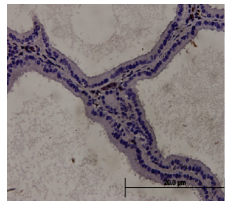

Normal

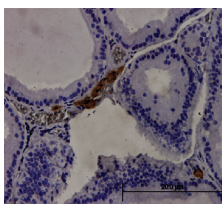

$\mathrm{BPH}$

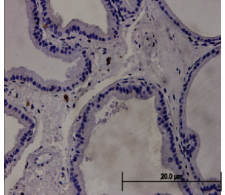

Ginkgo

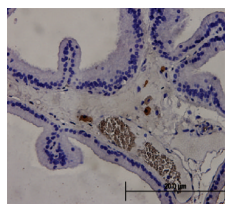

$\mathrm{BPH}+$ Ginkgo

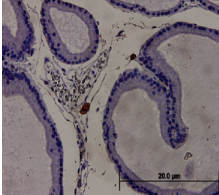

Ex

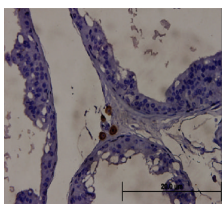

$\mathrm{BPH}+\mathrm{Ex}$

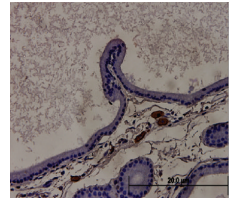

Ginkgo + Ex

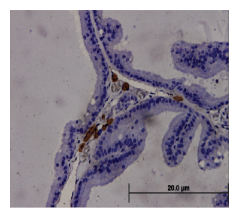

$\mathrm{BPH}+$ Ginkgo +

Ex

(a)

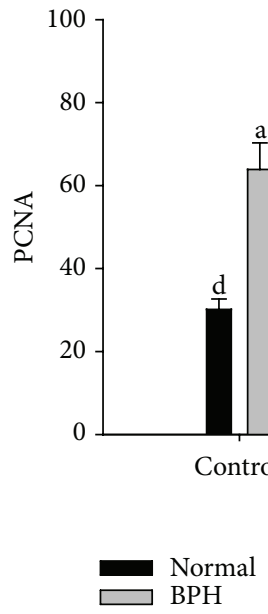

(b)

FIGURE 8: Immunohistochemical stain of the PCNA (a) $(\times 400)$ and its quantification (b). Data were collected and statistically treated with ANOVA and Duncan's multiple range tests $(n=3)$. Different letters indicate significant difference between groups $(P<0.05)$. PCNA: mouse proliferating cell nuclear antigen.

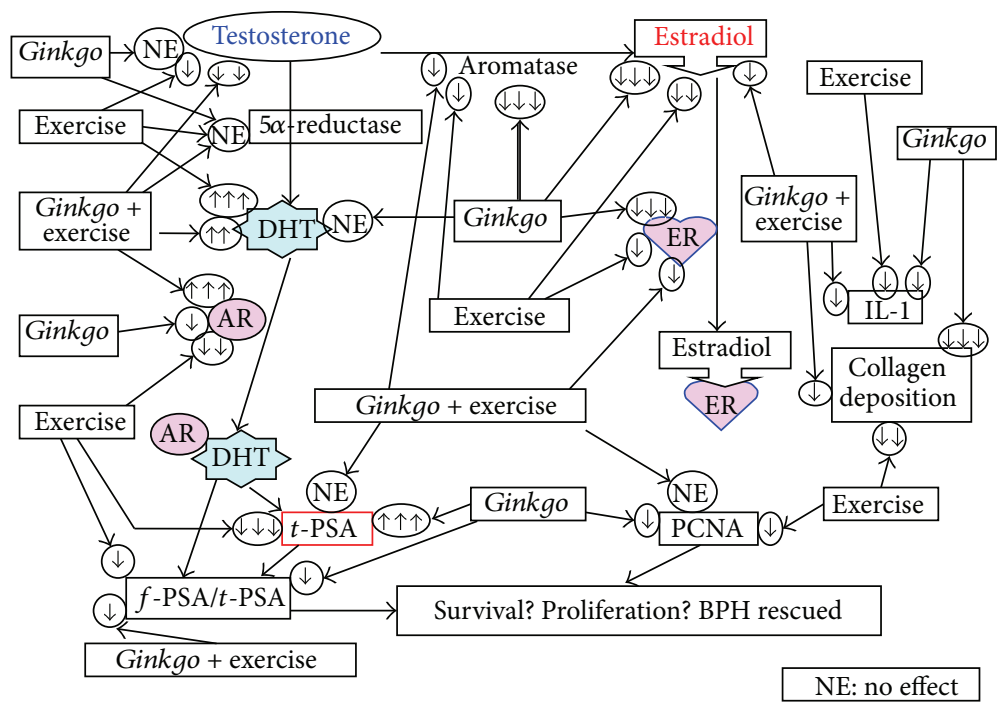

FIGURE 9: Summary of the therapeutic effects assessed from different treatments: Gingko, Ex, and the combined Gingko + Ex on the BPH. AR: androgen receptor, ER: estrogen receptor, $f$-PSA: free PSA, $t$-PSA: total PSA, PCNA: proliferating cell nuclear antigen. 
level). Exercise acts as an androgenic (regarding the DHT level), an anti-AR, a moderate anti-ER, a moderate aromatase inhibitor, and an estrogenic (regarding the estradiol level). The combined therapy behaves as an androgenic (regarding the DHT level), an AR-upregulator, a moderate anti-ER, a moderate aromatase inhibitor, and an antiestrogenic (regarding the estradiol level). Thus, the therapeutic outcomes could be very complicated and different from each other. Overall, pathologically Ginkgo alone and exercise alone may be more beneficial than the combined therapy.

\section{Conflict of Interests}

The authors report no conflict of interests.

\section{Acknowledgments}

The authors want to show their gratitude to the financial supports issued by Grant no. NSC 99-2320-B-038-011-MY3 and NSC 101-2320-B-038-030 from the National Science Council and by Grant no. SKH-TMU-93-37 from Shin Kong Wu Ho-Su Memorial Hospital.

\section{References}

[1] K. T. McVary, "BPH: epidemiology and comorbidities," American Journal of Managed Care, vol. 12, no. 5, supplement, pp. S122-S128, 2006.

[2] T. M. Nicholson and W. A. Ricke, "Androgens and estrogens in benign prostatic hyperplasia: past, present and future," Differentiation, vol. 82, no. 4-5, pp. 184-199, 2011.

[3] J. McNeal, "Pathology of benign prostatic hyperplasia. Insight into etiology," Urologic Clinics of North America, vol. 17, no. 3 , pp. 477-486, 1990.

[4] G. Untergasser, S. Madersbacher, and P. Berger, "Benign prostatic hyperplasia: age-related tissue-remodeling," Experimental Gerontology, vol. 40, no. 3, pp. 121-128, 2005.

[5] M. S. Lucia and J. R. Lambert, "Growth factors in benign prostatic hyperplasia: basic science implications," Current Urology Reports, vol. 9, no. 4, pp. 272-278, 2008.

[6] A. Sciarra, G. Mariotti, S. Salciccia et al., "Prostate growth and inflammation," Journal of Steroid Biochemistry and Molecular Biology, vol. 108, no. 3-5, pp. 254-260, 2008.

[7] C. K. M. Ho and F. K. Habib, "Estrogen and androgen signaling in the pathogenesis of BPH," Nature Reviews Urology, vol. 8, no. 1, pp. 29-41, 2011.

[8] T. Tsurusaki, D. Aoki, H. Kanetake et al., "Zone-dependent expression of estrogen receptors $\alpha$ and $\beta$ in human benign prostatic hyperplasia," The Journal of Clinical Endocrinology and Metabolism, vol. 88, no. 3, pp. 1333-1340, 2003.

[9] P. Alonso-Magdalena, C. Brössner, A. Reiner et al., "A role for epithelial-mesenchymal transition in the etiology of benign prostatic hyperplasia," Proceedings of the National Academy of Sciences of the United States of America, vol. 106, no. 8, pp. 28592863, 2009.

[10] S. Y. Lin and H. P. Chang, "Induction of superoxide dismutase and catalase activity in different rat tissues and protection from UVB irradiation after topical application of Ginkgo biloba extracts," Methods and Findings in Experimental and Clinical Pharmacology, vol. 19, no. 6, pp. 367-371, 1997.
[11] A. P. Berger, K. Kofler, J. Bektic et al., "Increased growth factor production in a human prostatic stromal cell culture model caused by hypoxia," Prostate, vol. 57, no. 1, pp. 57-65, 2003.

[12] G. Kramer, D. Mitteregger, and M. Marberger, "Is benign prostatic hyperplasia $(\mathrm{BPH})$ an immune inflammatory disease?" European Urology, vol. 51, no. 5, pp. 1202-1216, 2007.

[13] G. Novara, A. Galfano, V. Ficarra, and W. Artibani, "Anticholinergic drugs in patients with bladder outlet obstruction and lower urinary tract symptoms: a systematic review," European Urology, vol. 50, no. 4, pp. 675-683, 2006.

[14] N. B. Delongchamps, G. de la Roza, V. Chandan et al., "Evaluation of prostatitis in autopsied prostates-is chronic inflammation more associated with benign prostatic hyperplasia or cancer?" Journal of Urology, vol. 179, no. 5, pp. 1736-1740, 2008.

[15] J. C. Nickel, C. G. Roehrborn, M. P. O’Leary, D. G. Bostwick, M. C. Somerville, and R. S. Rittmaster, "Examination of the relationship between symptoms of prostatitis and histological inflammation: baseline data from the REDUCE chemoprevention trial," Journal of Urology, vol. 178, no. 3, pp. 896-901, 2007.

[16] A. Alcaraz, P. Hammerer, A. Tubaro, F. H. Schröder, and R. Castro, "Is there evidence of a relationship between benign prostatic hyperplasia and prostate cancer? Findings of a literature review," European Urology, vol. 55, no. 4, pp. 864-873, 2009.

[17] C. G. Roehrborn, J. Barkin, P. Siami et al., "Clinical outcomes after combined therapy with dutasteride plus tamsulosin or either monotherapy in men with benign prostatic hyperplasia $(\mathrm{BPH})$ by baseline characteristics: 4-Year results from the randomized, double-blind Combination of Avodart and Tamsulosin (CombAT) trial," Journal of the British Association of Urological Surgeons, vol. 107, no. 6, pp. 946-954, 2011.

[18] G. S. Gerber, "Phytotherapy for benign prostatic hyperplasia," Current Urology Reports, vol. 3, no. 4, pp. 285-291, 2002.

[19] J. A. Thomas, "Diet, micronutrients, and the prostate gland," Nutrition Reviews, vol. 57, no. 4, pp. 95-103, 1999.

[20] B. Singh, P. Kaur, Gopichand, R. D. Singh, and P. S. Ahuja, "Biology and chemistry of Ginkgo biloba," Fitoterapia, vol. 79, no. 6, pp. 401-418, 2008.

[21] K. Gohil, R. K. Moy, S. Farzin, J. J. Maguire, and L. Packer, "MRNA expression profile of a human cancer cell line in response to Ginkgo biloba extract: induction of antioxidant response and the golgi system," Free Radical Research, vol. 33, no. 6, pp. 831-849, 2000.

[22] R. Bridi, F. P. Crossetti, V. M. Steffen, and A. T. Henriques, “The antioxidant activity of standardized extract of Ginkgo biloba (EGb 761) in rats," Phytotherapy Research, vol. 15, no. 5, pp. 449$451,2001$.

[23] J. X. Chen, H. Zeng, X. Chen, C. Y. Su, and C. C. Lai, "Induction of oxygenase-1 by Ginkgo biloba extract but not its terpenoids partially mediated its protective effect against lysophosphatidylcholine-induced damage," Pharmacological Research, vol. 43, no. 1, pp. 63-69, 2001.

[24] M. Ahmad, S. Saleem, A. S. Ahmad et al., "Ginkgo biloba affords dose-dependent protection against 6-hydroxydopamineinduced parkinsonism in rats: neurobehavioural, neurochemical and immunohistochemical evidences," Journal of Neurochemistry, vol. 93, no. 1, pp. 94-104, 2005.

[25] F. V. DeFeudis, "A brief history of EGb 761 and its therapeutic uses," Pharmacopsychiatry, vol. 36, supplement 1, pp. S2-S7, 2003.

[26] G. R. Shah, M. V. Chaudhari, S. B. Patankar et al., "Evaluation of a multi-herb supplement for erectile dysfunction: a randomized 
double-blind, placebo-controlled study," BMC Complementary and Alternative Medicine, vol. 12, pp. 155-163, 2012.

[27] J. Sea, K. S. Poon, and K. T. McVary, "Review of exercise and the risk of benign prostatic hyperplasia," The Physician and Sportsmedicine, vol. 37, no. 4, pp. 75-83, 2009.

[28] P. T. Williams, "Effects of running distance and performance on incident benign prostatic hyperplasia," Medicine and Science in Sports and Exercise, vol. 40, no. 10, pp. 1733-1739, 2008.

[29] K. Suzuki, Y. Takezawa, T. Suzuki, S. Honma, and H. Yamanaka, "Synergistic effects of estrogen with androgen on the prostateeffects of estrogen on the prostate of androgen-administered rats and 5-alpha-reductase activity," Prostate, vol. 25, no. 4, pp. 169-176, 1994.

[30] K. C. Chen, C. L. Hsieh, C. C. Peng, and R. Y. Peng, "Exercise rescued chronic kidney disease by attenuating cardiac hypertrophy through the cardiotrophin- $\rightarrow$ LIFR/gp $130 \rightarrow$ JAK/STAT3 pathway," European Journal of Preventive Cardiology, 2012.

[31] J. S. Chen, Y. H. Chen, P. H. Huang et al., "Ginkgo biloba extract reduces high-glucose-induced endothelial adhesion by inhibiting the redox-dependent interleukin-6 pathways," Cardiovascular Diabetology, vol. 11, p. 49, 2012.

[32] J. Ma, M. Gharaee-Kermani, L. Kunju et al., "Prostatic fibrosis is associated with lower urinary tract symptoms," Journal of Urology, vol. 188, no. 4, pp. 1375-1381, 2012.

[33] C. Ulman, G. Buyukyazi, F. Taneli, and B. S. Uyanik, "Recreational and master athletic activity does not affect free and total prostate-specific antigen levels but lowers the free-to-total prostate-specific antigen ratio," Journal of International Medical Research, vol. 32, no. 6, pp. 583-589, 2004.

[34] S. M. Oh and K. H. Chung, "Antiestrogenic activities of Ginkgo biloba extracts," Journal of Steroid Biochemistry and Molecular Biology, vol. 100, no. 4-5, pp. 167-176, 2006.

[35] D. Giri and M. Ittmann, "Interleukin- $1 \alpha$ is a paracrine inducer of FGF7, a key epithelial growth factor in benign prostatic hyperplasia," American Journal of Pathology, vol. 157, no. 1, pp. 249-255, 2000.

[36] F. Deng, F. Gu, and T. Xia, "Proliferation and apoptosis status in benign prostatic hyperplasia," Zhonghua Wai Ke Za Zhi, vol. 34, no. 10 , pp. 620-622, 1996. 


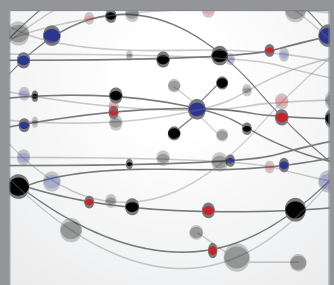

The Scientific World Journal
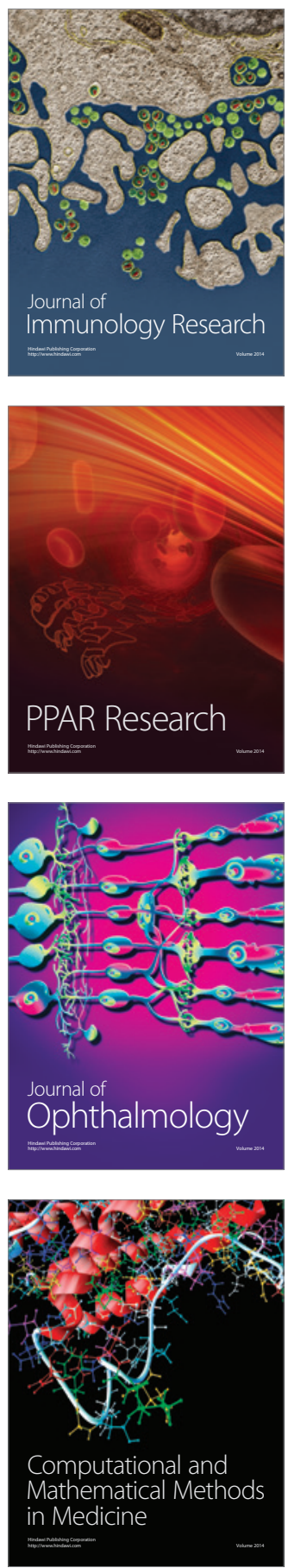

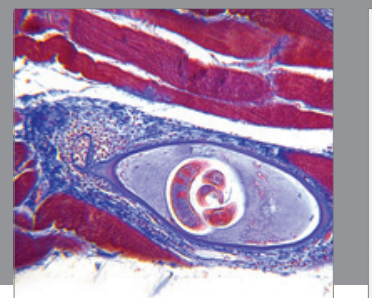

Gastroenterology

Research and Practice
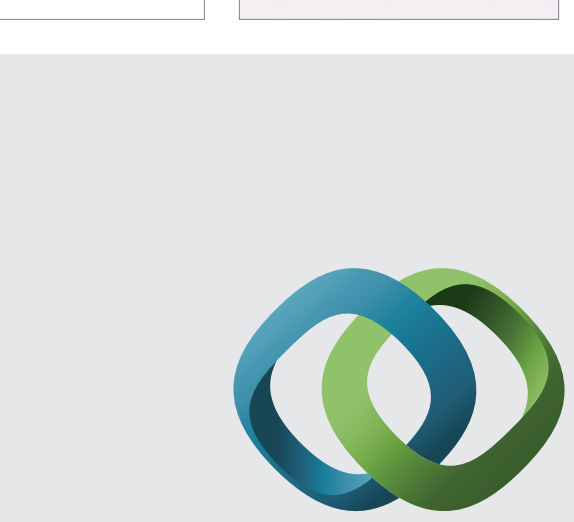

\section{Hindawi}

Submit your manuscripts at

http://www.hindawi.com
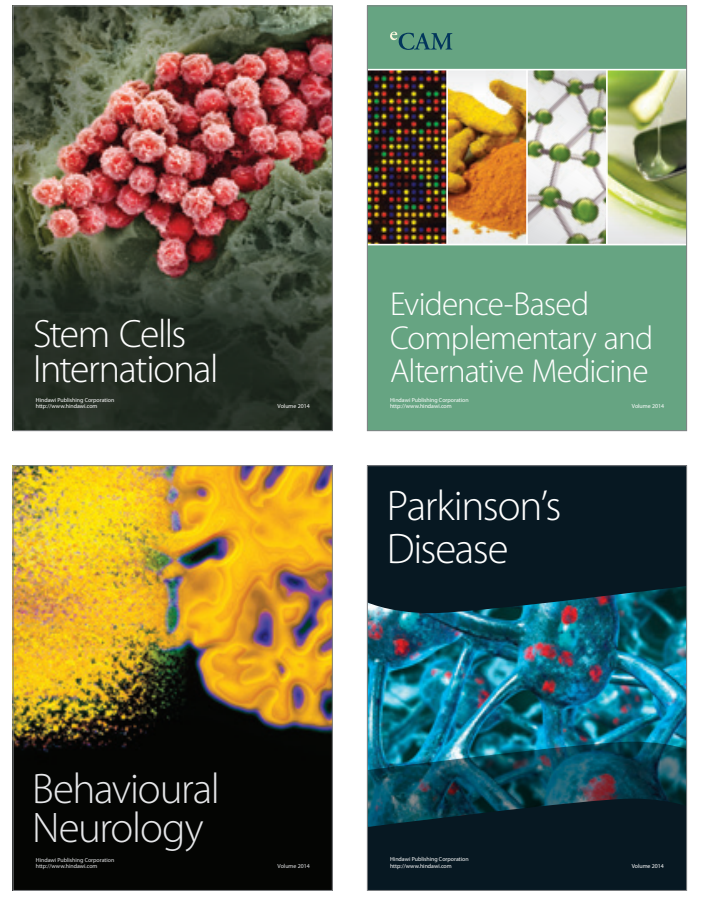
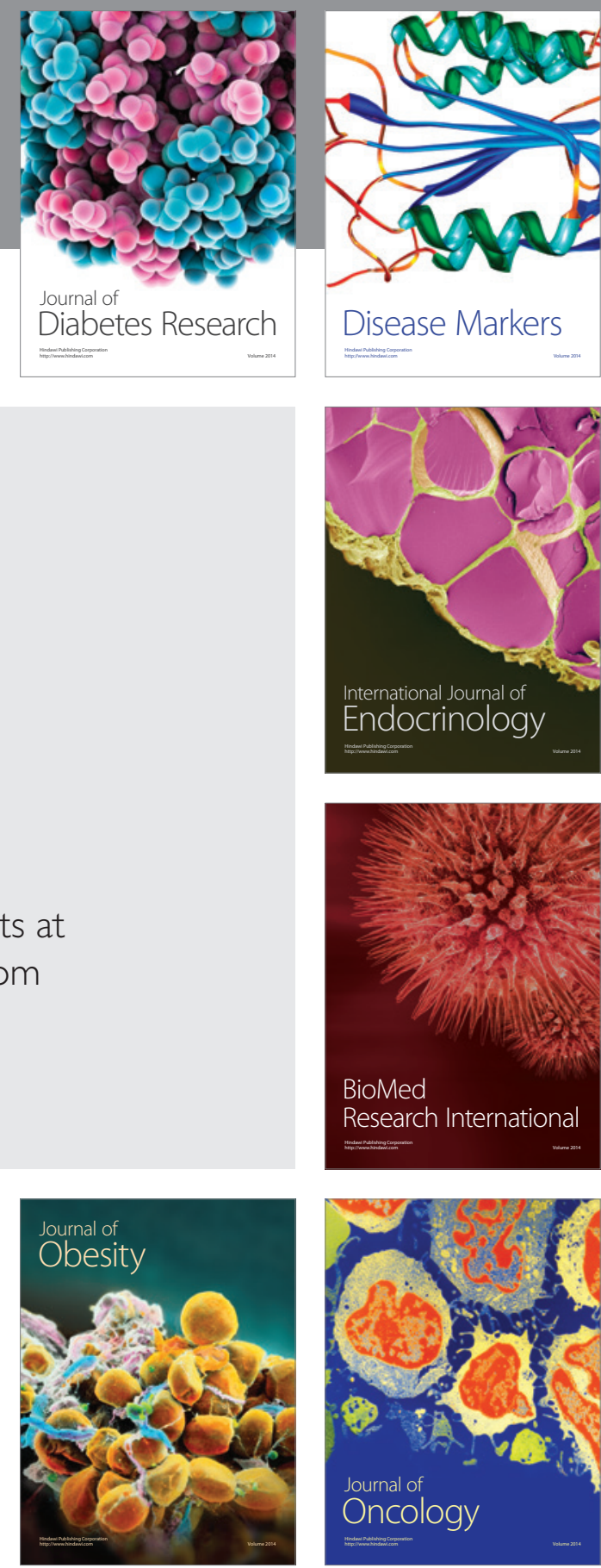

Disease Markers
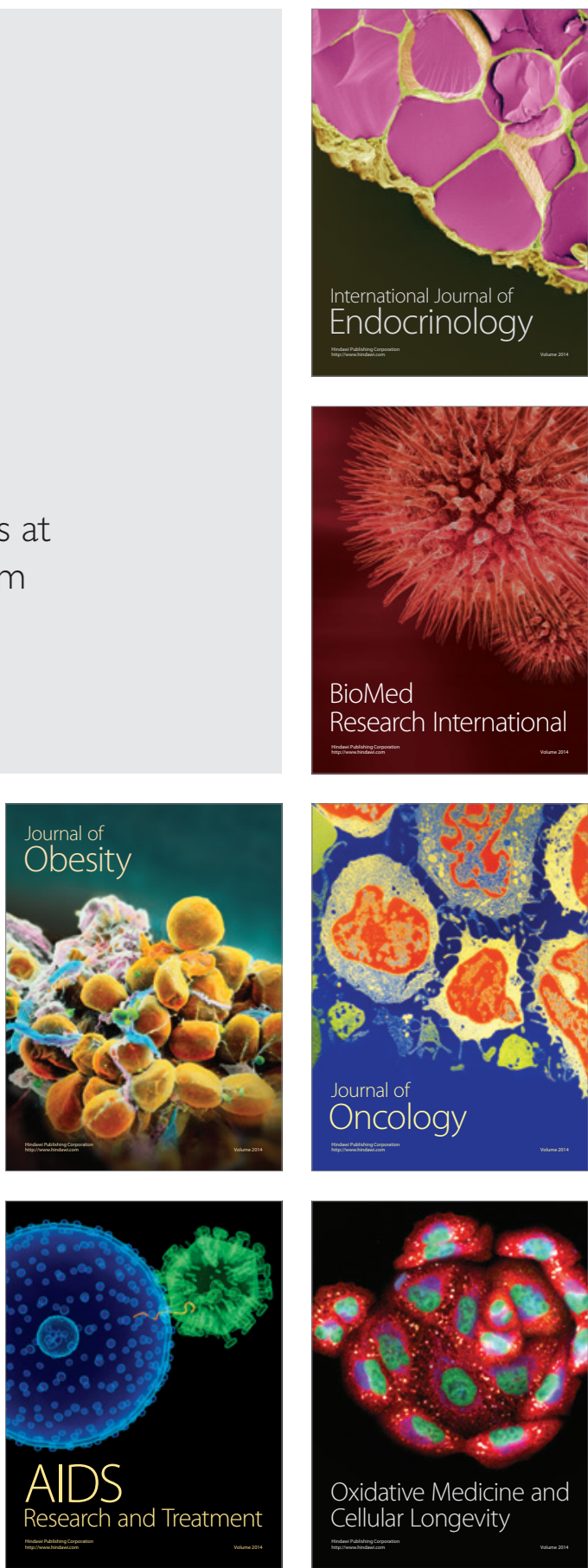